\title{
A giant pedunculated leiomyoma with cystic degeneration mimicking an ovarian malignancy
}

\author{
Shanthadevi Sambath*, Thangamani Balasubramanian
}

Department of Obstetrics \& Gynaecology, Government Theni Medical College, Theni, Tamilnadu, India

Received: 07 November 2015

Revised: 23 November 2015

Accepted: 15 December 2015

\section{*Correspondence:}

Dr. Shanthadevi Sambath,

E-mail: devisambath@gmail.com

Copyright: $(\odot$ the author(s), publisher and licensee Medip Academy. This is an open-access article distributed under the terms of the Creative Commons Attribution Non-Commercial License, which permits unrestricted non-commercial use, distribution, and reproduction in any medium, provided the original work is properly cited.

\begin{abstract}
Leiomyoma of the uterus is the most common type of tumor affecting the female pelvis and arises from uterine smooth muscle. The size of leiomyomas varies from microscopic to giant; giant myomas are exceedingly rare. We report an unusual case of a large, cystic, pedunculated uterine leiomyoma mimicking a primary malignant ovarian tumor on sonography and CT. A 41-year-old nulliparous woman presented with a history of lower abdominal pain 20 days and distension for a period of approximately 10 years. The patient's personal history revealed difficulty in walking, tiredness and sonography and CT examination showed a large mass that filled the abdomen. A preoperative diagnosis of a primary malignant ovarian tumor was made. The patient underwent laparotomy, total hysterectomy and bilateral salpingo-oopharectomy. The histology revealed a leiomyoma with extensive cystic degeneration. 50\% uterine fibroids are asymptomatic. As leiomyomas enlarge, they can outgrow their blood supply, resulting in various types of degeneration. Hyalinization is the most common type of degeneration, occurring in up to $60 \%$ of cases. Cystic degeneration, observed in about $4 \%$ of leiomyomas, may be considered extreme sequelae of edema. Typical appearances of leiomyomas are easily recognized on imaging. However, the atypical appearances that follow degenerative changes can cause confusion in diagnosis. Pedunculated leiomyomas should be considered in the differential diagnosis of a multilocular and predominantly cystic adnexal mass.
\end{abstract}

Keywords: Uterine leiomyoma, Giant myoma, Cystic degeneration

\section{INTRODUCTION}

Leiomyoma of the uterus is the most common tumor of the female pelvis which arises from uterine smooth muscle. ${ }^{1-3}$ Leiomyomas is found in nearly half of women over age 35 ; the prevalence increases during reproductive age and decreases after menopause. ${ }^{1}$ The size of leiomyoma varies from microscopic to giant fibroid. Giant leiomyoma with cystic degeneration is an exceedingly rare condition. It may sometimes present with clinical and imaging findings giving impression of other diagnosis like ovarian mass where final diagnosis depends on laparotomy findings with histological confirmation. Appropriate surgical management and careful perioperative care are necessary to obtain a good result after removal. Here, we present a case of a woman with giant pedunculated uterine leiomyoma that had undergone cystic degeneration, mimicking an ovarian tumor.

\section{CASE REPORT}

A 44-year-old unmarried woman presented with a history of lower abdominal pain 20 days and abdominal distension which was increased progressively over a period of approximately 10 years. Her menstrual cycles were regular. On examination she was thin built, a huge mass occupying the entire abdomen and it was cystic in nature. USG showed huge cystic mass suggestive of large ovarian cyst. CT abdomen showed a huge thick walled 
complex cystic mass with internal septation and multiple solid components in the dependent part of the cyst, the mass filled the whole abdomen, and measures about $35 \mathrm{~cm}$ vertically, and $45 \mathrm{~cm}$ horizontally, uterus was compressed by the mass. CA 125 value was $136.4 \mathrm{u} / \mathrm{ml}$, and the other investigations were normal. A preoperative diagnosis of a malignant ovarian tumor was made. Laparotomy was done, the cystic mass filling the entire abdomen was delivered out. Bowel and omentum were stretched over the mass and adherent. After the adhesions were released the mass was found to arise from the fundus of the uterus with a pedicle, both ovaries were normal. Total hysterectomy and bilateral salping oophorectomy was done. During surgery patient developed severe hypotension which was managed effectively with ionotropes, adrenaline and blood. Four units of blood was transfused. Postoperatively she was managed in the intensive care unit and she was discharged in good condition after 15 days.

The weight of the mass was $18 \mathrm{~kg}$ and contains brownish fluid with solid part in the lower part of the mass. The histopathology of the mass revealed a leiomyoma with extensive cystic degeneration. The final diagnosis was giant pedunculated uterine leiomyoma with marked cystic degeneration.

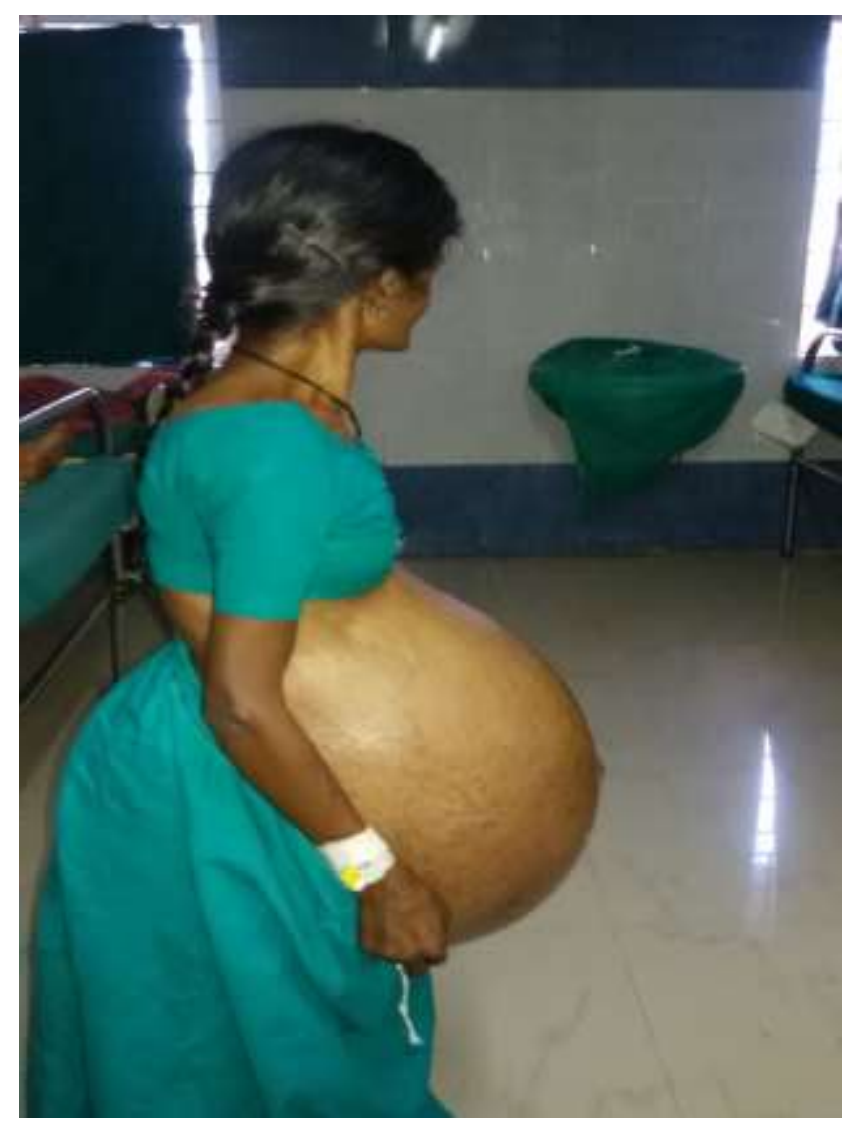

Figure 1: Patient with huge tumor.

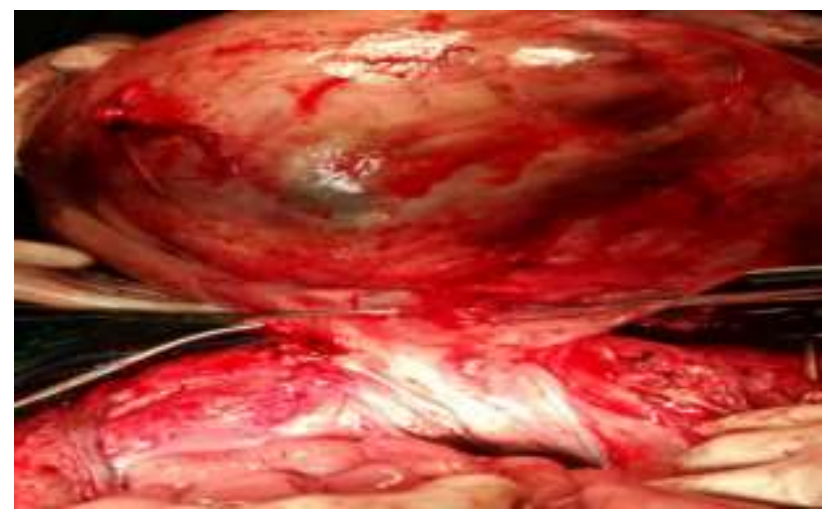

Figure 2: Laparatomy showing tumor arising from fundus.

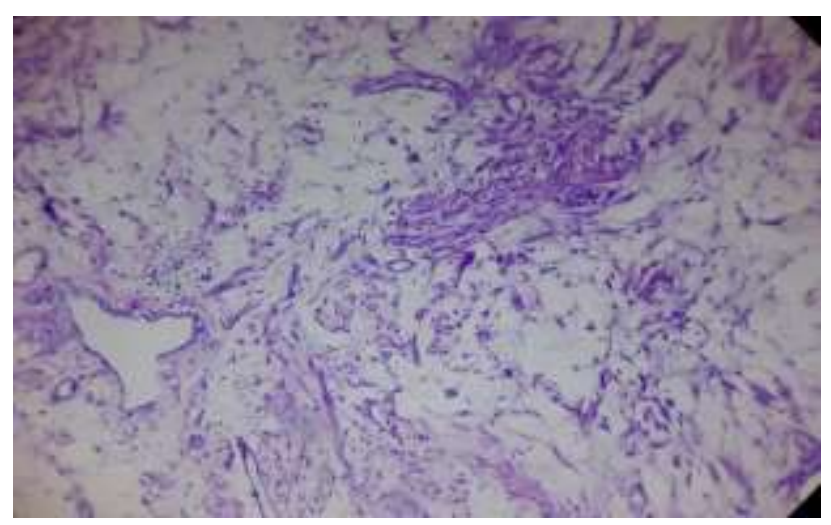

Figure 3: Fibroid with cystic degeneration.

\section{DISCUSSION}

Leiomyoma is the most common uterine tumour, composed of smooth muscle with varying amount of fibrous connective tissue. Large uterine fibroids can cause pain, AUB and presser symptom. They can also affect reproduction by causing infertility, miscarriage and/or premature labor. Subserosal fibroids may be pedunculated and simulates ovarian neoplasm.

Only few cases of giant uterine tumors have been reported in the recent literature. The potential for uterine leiomyomas to grow to an extreme size before causing symptoms is quite remarkable. This is likely due to the relatively large volume of the abdominal cavity, the distensibility of the abdominal wall and the slow growth rate of these tumors.

As the fibroids enlarge they may outgrow their blood supply and undergo various types of degeneration. ${ }^{1}$ Hyaline degeneration is the most common type occurring in about $60 \%$ of cases, cystic degeneration is rare occur in $4 \%$ of cases and considered an extreme sequelae of edema. ${ }^{1}$

Ultrasonography is the preferred method for the initial evaluation of fibroids because it is non-invasive and the most cost-effective. The relative echogenicity of 
leiomyomas depends on the ratio of fibrous tissue to smooth muscle, the extent of degeneration and the presence of calcification. However, cystic degenerating fibroids can be mistaken for other clinical entities and can present a diagnostic challenge. ${ }^{3,5}$ These areas of cystic degeneration are irregular and can appear as a combination of cystic and solid components of variable echogenicity. In the literature, there have been reports of cystic uterine fibroids mimicking endometrioma and large ovarian masses. ${ }^{2,4}$ A CT scan can be useful; however, leiomyomas with cystic degeneration are indistinguishable from malignant ovarian tumour with presence of both cystic and solid areas as happened in our case. MRI can define the uterine and ovarian masses, but the availability and high cost are serious limitations. ${ }^{3}$

The management of uterine fibroids may be expectant management, myomectomy, uterine artery embolization, or hysterectomy depending on various factors, including age, type of fibroids and severity of symptoms, desire for fertility. A surgical approach is most frequently preferred for management of giant leiomyoma. ${ }^{3}$ During surgery the position of the patient, type of anaesthesia, and the incision should be considered as important in the management of giant leiomyoma and the multidisciplinary care in the post-operative period gives good outcome.

\section{CONCLUSIONS}

Although fibroids typically have a characteristic USG appearance, degenerating fibroids can have variable patterns and pose diagnostic challenges. ${ }^{3}$ This case represents an unusual case of a huge cystic leiomyoma without any symptom. Pedunculated leiomyomas with cystic degeneration should be considered in the differential diagnosis of a multilocular and predominantly cystic adnexal mass.

Funding: No funding sources

Conflict of interest: None declared

Ethical approval: Not required

\section{REFERENCES}

1. Aydin C, Eris S, Yakin Y, Yalcin Y, Selim HS. A giant cystic fibroid mimicking ovarian malignancy. Int J Surg case Rep. 2013;4(11):1010-2.

2. Pandy D, Priyadarshini P, Feroz MS, Roopa PS, Kudva R, Shetty J, et al. Massive degenerated leiomyomas masquerading ovarian neoplasm. Sri Lanka Journal of Obstetrics and Gynaecology. 2011;33:163-5.

3. Kaushik C, Prasad A, Singh Y, Baruah BP. Case series: cystic degeneration in uterine fibroid. Indian $\mathrm{J}$ Radiol Imaging. 2008;18(1):69.

4. Reddy NM, Jain KA, Gerscovich EO. A degenerating cystic uterine fibroid mimicking endometrioma on sonography. American Institute of Ultrasound in Medicine (aium), the medical ultrasound journal. 2003;22(9):973-6.

5. Low SCA, Chong CL. A case of cystic leiomyoma mimicking an ovarian malignancy. Ann Acad Med Singapore. 2004;33:371-4.

Cite this article as: Sambath $\mathrm{S}$, Balasubramanian $\mathrm{T}$. A giant pedunculated leiomyoma with cystic degeneration mimicking an ovarian malignancy. Int J Reprod Contracept Obstet Gynecol 2016;5:252-4. 